\title{
Establishment of a murine breast tumor model by subcutaneous or orthotopic implantation
}

\author{
YI ZHANG, GAN-LIN ZHANG, XU SUN, KE-XIN CAO, CONG MA, NAN NAN, \\ GUO-WANG YANG, MING-WEI YU and XIAO-MIN WANG \\ Department of Oncology, Beijing Hospital of Traditional Chinese Medicine, \\ Capital Medical University, Beijing 100010, P.R. China
}

Received May 21, 2016; Accepted January 10, 2018

DOI: $10.3892 / \mathrm{ol} .2018 .8113$

\begin{abstract}
A number of murine models are used to mimic the pathology of breast cancer. Tissue inoculation and cell inoculation using orthotopic implantation (OS) and subcutaneous implantation (SQ) are commonly used to generate murine models to investigate cancer. However, limited information is available in regard to the variations of these methods. The present study compared growth, metastasis, survival and histopathology of tumors produced using OS and SQ to characterize features of the tumors produced by the two distinct methods. Additionally, the present study aimed at providing increased options for investigators when designing experiments. 4T1-luc2 cell suspension or 4T1-luc2 tissue suspension was inoculated using either OS or SQ into BALB/c mice. Tumor growth and metastasis were detected using an in vivo imaging system and calipers. Excised tumors and lung were assessed by tissue staining with hematoxylin and eosin, and the vessel marker cluster of differentiation 31 . The results of the present study revealed that the cell suspension generated breast tumors of increased size, which was visualized and determined, following inoculation, using calipers at an earlier time point compared with tumors produced by tissue suspension. The increasing bioluminescent trend of OS tumors was more marked compared with that of SQ tumors. The volume of OS tumor was increased with decreased variation, compared with that of SQ tumors. In addition, the OS tumor exhibited increased microvessel density. Bioluminescent signals and histological results in regard to metastasis were consistent: OS implantation produced increased lung metastasis compared with that of SQ implantation, although they exhibited similar survival times. The results of the present
\end{abstract}

Correspondence to: Dr Xiao-Min Wang or Dr Gan-Lin Zhang, Department of Oncology, Beijing Hospital of Traditional Chinese Medicine, Capital Medical University, 23 Back Road of Art Gallery, Beijing 100010, P.R. China

E-mail: wangxiaomin_bhtcm@126.com

E-mail: kalinezhang@163.com

Key words: breast tumor, animal model, subcutaneous implantation, orthotopic implantation, lung metastasis study indicated that the inocula from distinct sources (tissue or cell) affected tumor growth. Furthermore, breast tumor progression and histopathological characteristics were distinct between $\mathrm{OS}$ and $\mathrm{SQ}$, whereas OS exhibited increased malignant behavior. Understanding the characteristics of murine breast cancer models established by diverse methods may aid investigators to select appropriate animal models, according to the requirements of the study.

\section{Introduction}

Breast cancer is the most frequently diagnosed cancer and is the leading cause of female mortality worldwide, accounting for $25 \%$ of the total number of cancer cases and $15 \%$ of all cancer-associated female mortality (1). The incidence of breast cancer is still progressively increasing (2). Therefore, females with breast cancer represent a major public health priority and has received hundreds of billions of US dollars over the last 20 years to fund studies and drug development (3). In order to bridge the gap between preclinical and clinical studies, murine models are broadly used to mimic the development of breast cancer and assess the efficacy of therapeutic strategies. It is evident that no single murine model is able to replicate the process of cancer progression $(4,5)$, therefore it is important to choose a relevant and appropriate model.

Murine models of breast cancer currently used are: Xenograft models, syngeneic murine models (directly implanted, chemically or virally induced) and genetically engineered mice (GEM) (including transgenic and knockout methods) (6). GEM is generated to mimic human breast cancer by targeted expression of growth factors, receptors, proteases, oncogenes or tumor suppressor genes; this has the advantage of elucidating the roles that transgenes serve in breast tumorigenesis and the efficacy of targeted therapeutics (7-10). However, GEM typically takes several months to generate and has a high cost. Furthermore, transgenic models exhibit a low incidence of metastasis, thus it is not possible to investigate the underlying molecular mechanisms of cancer metastasis or metastasis-related therapies $(4,11)$.

Xenograft models serve a dominant role in screening drugs for safety and efficacy. This type of model is easy to operate and is widely utilized to study inhibitors of steroid receptor signaling and drug resistance $(12,13)$. However, the formation of solid tumors is influenced by the extent of mice 
immunodeficiency. Nude mice exhibit insufficiency of tumor growth and a low metastasis rate due to natural killer cell or B cell immune response $(14,15)$. Additionally, the tumor xenograft in immune-deficient mice obviates the tumor-host interaction, impairs the metastatic microenvironment and tends to generate false positive results $(16,17)$.

A syngeneic murine model, the most common type of model used for studying mechanisms of tumor growth and metastasis, is characterized by implanted cells or tissue of the same species or genotype as the host animal (18). Therefore, these models provide the context of an intact immune system and host stroma and extracellular matrix for evaluating the tumor-environment interaction, antitumor immune response and primary metastatic tumor relevance. In mammary tumor models, 4T1 or 4T1-luc models are useful tools for investigating antitumor and anti-metastatic effects of various drugs due to their high invasive nature $(5,19-22)$, making it a well-accepted model for diverse studies.

The selection of modeling methods varies in different studies, including tissue and cell inoculation, orthotopic implantation (OS), subcutaneous implantation (SQ) and tail vein injection (TV). As for the syngeneic model, the characteristic of tumor progression is related to the inocula, the implantation site and the number of inoculated cells $(4,5,16,20,23,24)$. Therefore, a good understanding of the characteristics of models induced by distinct methods will aid investigators to make appropriate choices, according to the research requirements. The aim of the present study was to compare distinct methods involving inocula and implantation site in mouse models, to provide reference information for preclinical studies; in particular, to elucidate the distinctions of growth, metastasis, survival and histopathology of tumors produced using OS and SQ.

\section{Materials and methods}

Chemicals and reagents. D-luciferin was purchased from Gold Biotechnology Inc. (St. Louis, MO, USA). The primary antibody against cluster of differentiation (CD) 31 (catalog no. ab28364) was purchased from Abcam (Cambridge, UK). Two-step histostaining reagent kit (PV-9000), and hematoxylin and eosin staining kit (ZLI-9615) were purchased from Beijing Zhong Shan Jinqiao Biotechnology Co., Ltd. (Beijing, China).

Preparation of cell and tissue suspension. The 4T1-luc2 murine breast cancer cell line (PerkinElmer, Inc., Waltham, MA, USA) was cultured in RPMI-1640 medium (Gibco ${ }^{\circledR}$; Thermo Fisher Scientific, Inc., Waltham, MA, USA), supplemented with $10 \%$ fetal bovine serum (Gibco ${ }^{\circledR}$; Thermo Fisher Scientific Inc.) and $1 \%$ penicillin-streptomycin (HyClone; GE Healthcare Life Sciences, Logan, UT, USA). Cells were suspended at a density of $1 \times 10^{4}$ cells $/ 25 \mu \mathrm{l}$. Tissue suspension was prepared as aforementioned, with modification $(25,26)$; the 4T1-luc2 breast tumor was ground into a single cell suspension and filtered through a $70 \mu \mathrm{m}$ disposable cell strainer (Thermo Fisher Scientific, Inc.) in PBS and finally suspended at a concentration of $1 \times 10^{4}$ cells $/ 25 \mu 1$.

Animal model. Female BALB/c mice ( $\mathrm{n}=20 ; 8$ weeks old; 18-20 g) were purchased from Vital River Laboratory Animal Technology Co. Ltd., (Beijing, China) and randomly assigned to each group $(n=10)$. Animals were housed at $22 \pm 5^{\circ} \mathrm{C}$ in a $12 \mathrm{~h}$ light/dark cycle and fed rodent chow and water freely. Orthotopic mammary fat pad implantation was performed as follows: Female BALB/c mice were inoculated with $25 \mu \mathrm{l}$ cell or tissue suspension $\left(1 \times 10^{4}\right)$ in the mammary fat pads under anesthesia via Matrx VMS anesthesia machine (Midmark Corporation, Dayton, OH, USA) by continuous inhalation of $2 \%$ isoflurane gas for 5-10 $\mathrm{min}$. Sterile tweezers were used to lift the fourth nipple and a syringe needle (BD Biosciences, San Jose, CA, USA) was used to implant cell or tissue suspensions directly into the mammary fat pad. For subcutaneous implantation, female $\mathrm{BALB} / \mathrm{c}$ mice were subcutaneously inoculated with $25 \mu \mathrm{l}$ cell suspension $\left(1 \times 10^{4}\right)$ under anesthesia by continuous inhalation of 2\% isoflurane gas for 5-10 min. The skin was tented up and the 4T1-luc2 cells were implanted under the skin in the dorsal flank regions. Tumor length (L) and width (W) were measured twice weekly using calipers, and tumor volume (V) was calculated as $\left[\mathrm{V}=\left(\mathrm{L}_{\mathrm{x} \mathrm{W}} \mathrm{W}^{2}\right) / 2\right]$. Bioluminescence images of primary tumor were captured using IVIS Spectrum (PerkinElmer, Inc.) $10 \mathrm{~min}$ after intraperitoneal injection of D-luciferin $(150 \mathrm{mg} / \mathrm{kg}, 200 \mu \mathrm{l})$. After $\sim 30$ days, the primary tumors were removed and incisions were sutured under isoflurane anesthesia. Subsequently, the lung metastasis was detected with bioluminescent imaging. Light outputs were quantified using Living Image (version 4.3; PerkinElmer, Inc.). At day 56, the mice were sacrificed following anesthetization by pentobarbital $(60 \mathrm{mg} / \mathrm{kg}$ intraperitoneal injection) and lung tissues were obtained for histology analysis. Humane endpoints for the present study were as follows: Body weight, which was monitored twice weekly. When the weight after tumor cell inoculation decreased by $>10 \%$ when compared with the initial weight of the mouse. Secondly, behavioral observations taken during the experiment. When the food/water consumption and the daily activity reduced by $50 \%$, or if the fur became dull. Thirdly, tumor length and width were measured twice weekly using digital calipers. When the volume of primary tumor reached $\sim 800 \mathrm{~mm}^{3}$, the mice were sacrificed. Also painkillers were administered following tumor resection. If mice appeared in pain even with following painkiller administration, the mice were sacrificed. If continuous bleeding and wound infection were observed, the mice were sacrificed. Finally, if the wounds fail to heal following tumor resection past 5 days, the mice were sacrificed. All animal experiments were approved by the Institution of Animal Care and Use Committee of Capital Medical University (ref. no. AEEI-2014-052).

Histology and immunohistochemistry. The breast tumors and lungs were selected for histological examination. Tissues were dissected, paraffin-embedded and sectioned (6 $\mu \mathrm{m}$ thick). Sections were stained with hematoxylin and eosin (H\&E). Anti-CD31 rabbit polyclonal antibody was used for immunohistochemical (IHC) staining of breast tumor and lung sections for microvessel density (MVD) analysis. IHC staining of CD31 was performed according to the manufacturer's protocol. Briefly, paraffin-embedded sections were deparaffinized with xylene and rehydrated in a graded ethanol series at room temperature, and endogenous peroxidase activity was quenched (30 min) by $3 \% \mathrm{H}_{2} \mathrm{O}_{2}$ in methanol. Sections were subsequently pretreated in boiling citrate buffer for $20 \mathrm{~min}$. Sections were blocked in $10 \%$ normal goat serum in PBS for $60 \mathrm{~min}$ and then incubated with primary antibodies (anti-CD31 rabbit polyclonal antibody, 

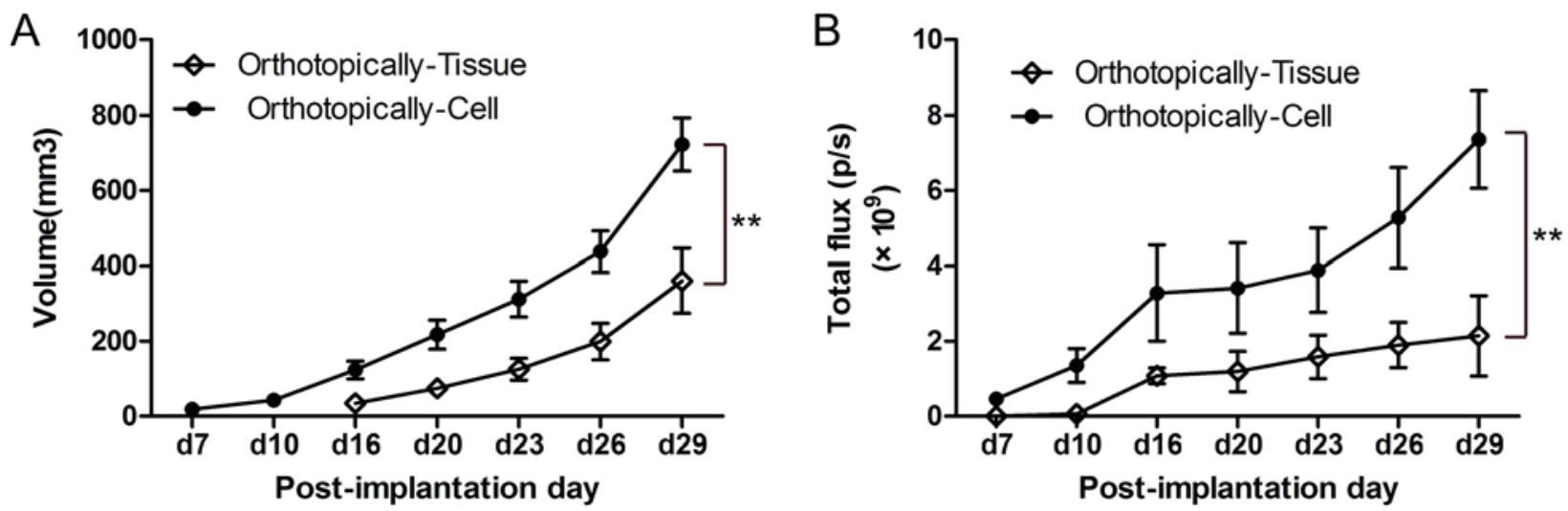

Figure 1. (A) Volume and (B) total flux of the primary tumor was measured from 7 days after implantation. Cultured cell suspension implantation generated an increased primary tumor which was visualized and determined using calipers at an earlier time point compared with tissue suspension implantation. The difference in total flux and volume between cell suspension and tissue were significant after analysis by repeated measures analysis of variance $\left({ }^{* * *} \mathrm{P}<0.01\right)$. p/s, photons/sec.

dilution 1:100) overnight at $4^{\circ} \mathrm{C}$. Following two washes in PBS, sections were incubated with $100 \mu$ l goat anti-rabbit secondary antibody from the two-step histostaining reagent kit (Beijing Zhong Shan Jinqiao Biotechnology Co., Ltd.) according to the manufacturer's protocol for $30 \mathrm{~min}$ at room temperature. The immunoreaction was visualized when brown precipitates formed following incubation in diaminobenzidine. Sections were subsequently washed with water and counterstained with $0.5 \%$ hematoxylin for $3 \mathrm{~min}$ at room temperature. Finally, sections were viewed using an upright light microscope (magnification, x200 or x100) (Olympus BX53; Olympus Corporation, Tokyo, Japan). MVD was determined in five fields with a higher density of $\mathrm{CD} 31^{+}$cells and cell clusters. The presence of visible blood vessel lumen was not required to be defined as positive (27).

Statistical analysis. Results are presented as the mean \pm standard deviation for individual experiments. Statistical significance was determined by Student's t-test or repeated measures analysis of variance followeed by Dunnett's test for multiple comparisons. Kaplan-Meier estimator survival statistical analysis was utilized for survival data. All calculations were performed using GraphPad Prism (version 5.0; GraphPad Software Inc., La Jolla, CA, USA). $\mathrm{P}<0.05$ was considered to indicate a statistically significant difference.

\section{Results}

Cell suspension generates larger breast tumor compared with tissue suspension. After 7 days of implantation, the tumor growth was determined using calipers and a bioluminescence imaging system. The cell suspension, visualized and determined at an earlier time point ( 7 days vs. 16 days; Fig1A), generated larger primary tumors compared with that of the tissue suspension $(722.81 \pm 70.77$ vs. $360.67 \pm 86.12 \mathrm{~mm}^{3}$ ). Bioluminescence imaging also revealed increased total flux of tumor in animals inoculated with the cell suspension $\left[(7.36 \pm 1.29) \times 10^{9}\right.$ vs. $(2.14 \pm 1.07)$ $\mathrm{x} 10^{9}$ photons/sec; Fig. 1B].

OS produces larger and less variable tumors with increased lung metastasis compared with SQ. The 4T1-luc2 cell suspension was implanted via OS or SQ in BALB/c mice. The tumor growth and metastasis were detected in vivo using an imaging system or calipers. The volume of tumor produced using OS implantation increased to $722.81 \mathrm{~mm}^{3}$ at day 29 , which was increased compared with that of SQ implantation $\left(447.18 \pm 145.20 \mathrm{~mm}^{3}\right)$. The variation in OS tumor volume was decreased compared with SQ (Fig. 2A). Furthermore, the tumor progression of OS and SQ was additionally analyzed by quantification of bioluminescent signals. The results of the present study demonstrate that the increased trend of total flux of OS tumor was significantly increased, compared with that of SQ tumor $\left[(7.36 \pm 1.29) \times 10^{9}\right.$ vs. $(3.14 \pm 1.04) \times 10^{9}$ photons/sec; Fig. 2B)]. Following resection of the primary tumor, bioluminescent signals of lung metastasis were determined. The results of the present study revealed that OS implantation induced increased lung metastasis, compared with that in SQ implantation (Fig. 3A), and the total flux of OS lung metastasis $\left(9.17 \times 10^{6}\right.$ photons/sec) increased by 1.8 times compared with SQ $\left(3.23 \times 10^{6}\right.$ photons/sec). Although OS produced tumors of increased size and increased lung metastasis compared with SQ, there was no difference in mass mortality between the OS and SQ groups (Fig. 3B).

Histology. Primary tumors and metastatic lungs were fixed in buffered formalin and embedded in paraffin. Subsequently, tissue sections were stained by $\mathrm{H} \& \mathrm{E}$ and probed with an anti-CD31 antibody. OS and SQ tumors presented as a solid mass comprising poorly differentiated cancer cells (Fig. 4A and B). Notably, OS primary tumors revealed increased tissue heterogeneity with the involvement of adipocytes. Quantification of MVD demonstrated a marked variation between the tumors collected from OS and SQ (Fig. 4C-E) as an increased number of blood vessels were observed in OS. Additionally, H\&E staining identified that OS and SQ produced isolated lung metastatic tumors (Fig. 5A-H). An increased number of metastatic tumor foci were visualized using microscopy in OS sections.

\section{Discussion}

It is critical to select a proper model to mimic the initiation and progression of breast cancer when evaluating the efficacy of antitumor drugs and it is considered the key to success in studies $(4,28)$. A number of murine models have become 
A

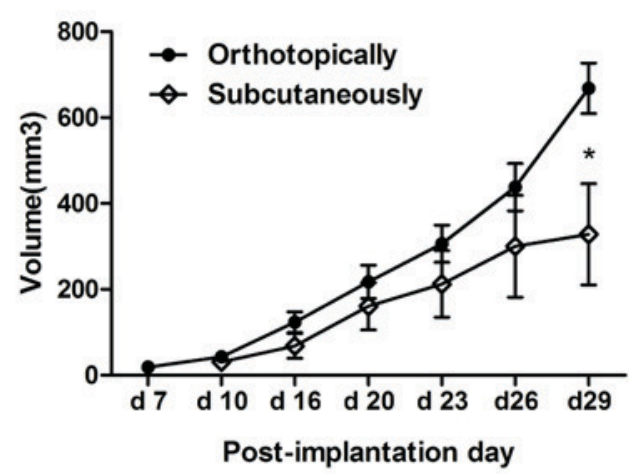

B

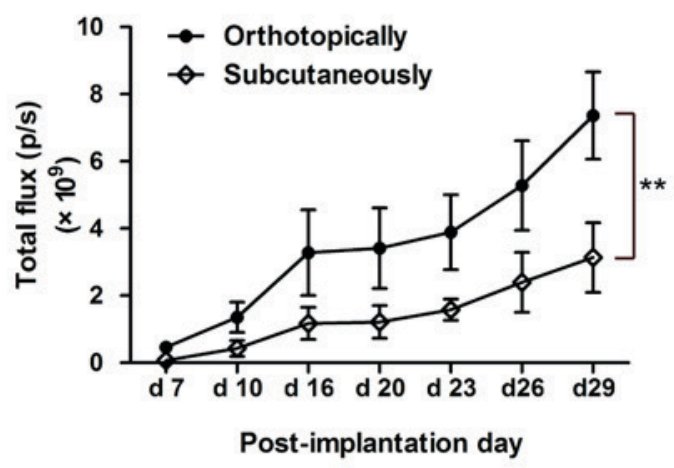

C
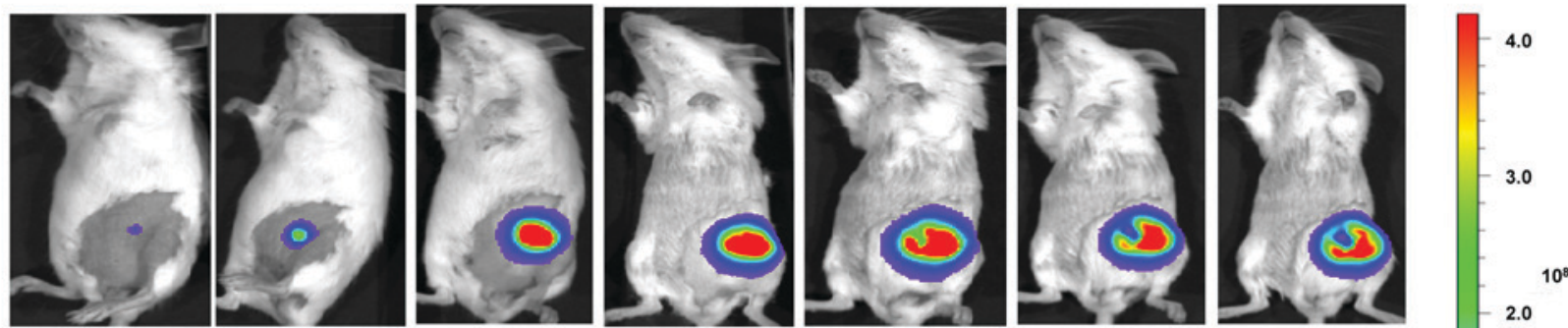

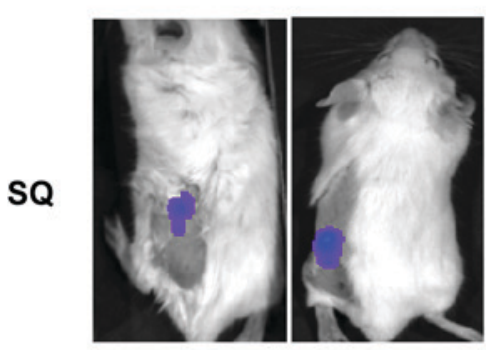

day 7

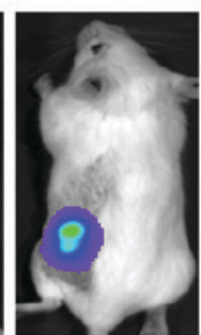

day 16

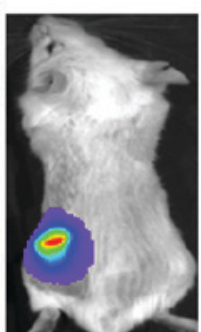

day 20

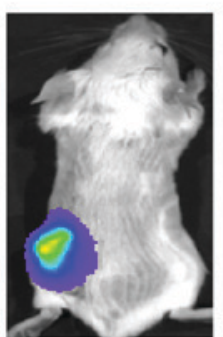

day 23

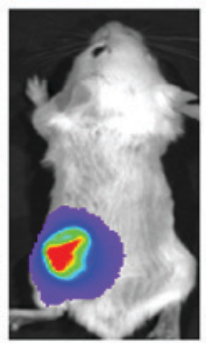

day 26

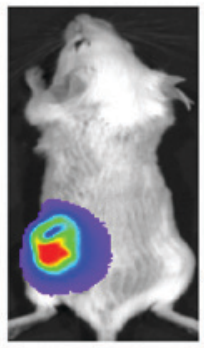

day 29

Figure 2. Comparison of different implantation sites. (A) Volume of primary tumors at orthotopic and subcutaneous site at different times. OS primary tumors were of increased size and decreased variability compared with that of SQ primary tumors. Significance was observed at day 29 ("P<0.05 vs. SQ). (B) Quantification of bioluminescent signals (photons/sec) at orthotopic and subcutaneous sites at various times. The increasing trend of OS primary tumors were more marked compared with that of SQ primary tumors $\left({ }^{* *} \mathrm{P}<0.01\right)$. (C) Bioluminescent images of primary tumors observed at various time points after day 7 of implantation.

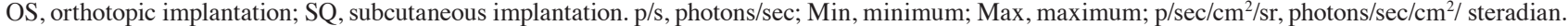

A

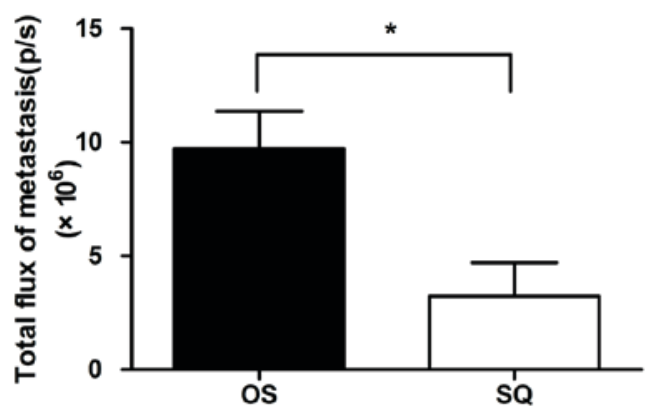

B

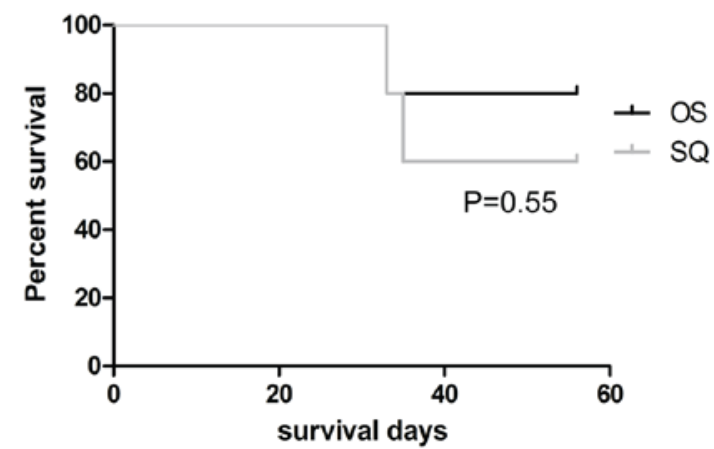

Figure 3. (A) Determination of bioluminescent signals to analyze the extent of lung metastasis following primary tumor resection. Total flux of OS lung metastasis was increased compared with that of SQ ( $\left.{ }^{*} \mathrm{P}<0.05\right)$. (B) Kaplan-Meier estimator analysis demonstrated there was no mass mortality in SQ and OS; however, SQ led to slightly decreased survival compared with that of OS $(\mathrm{P}=0.55)$. p/s, photons/second; OS, orthotopic implantation; SQ, subcutaneous implantation.

available during the last two decades and syngeneic breast cancer murine models, including the 4T1 series of murine mammary cancer models $(5,19-22,29)$, remain widely used, owing to its syngeneic property of cancer cells and experimental mice.
Animal models comprising distinct methods exhibit varied characteristic and application scope. Thus, understanding the advantages and limitations of these modeling methods is required. 

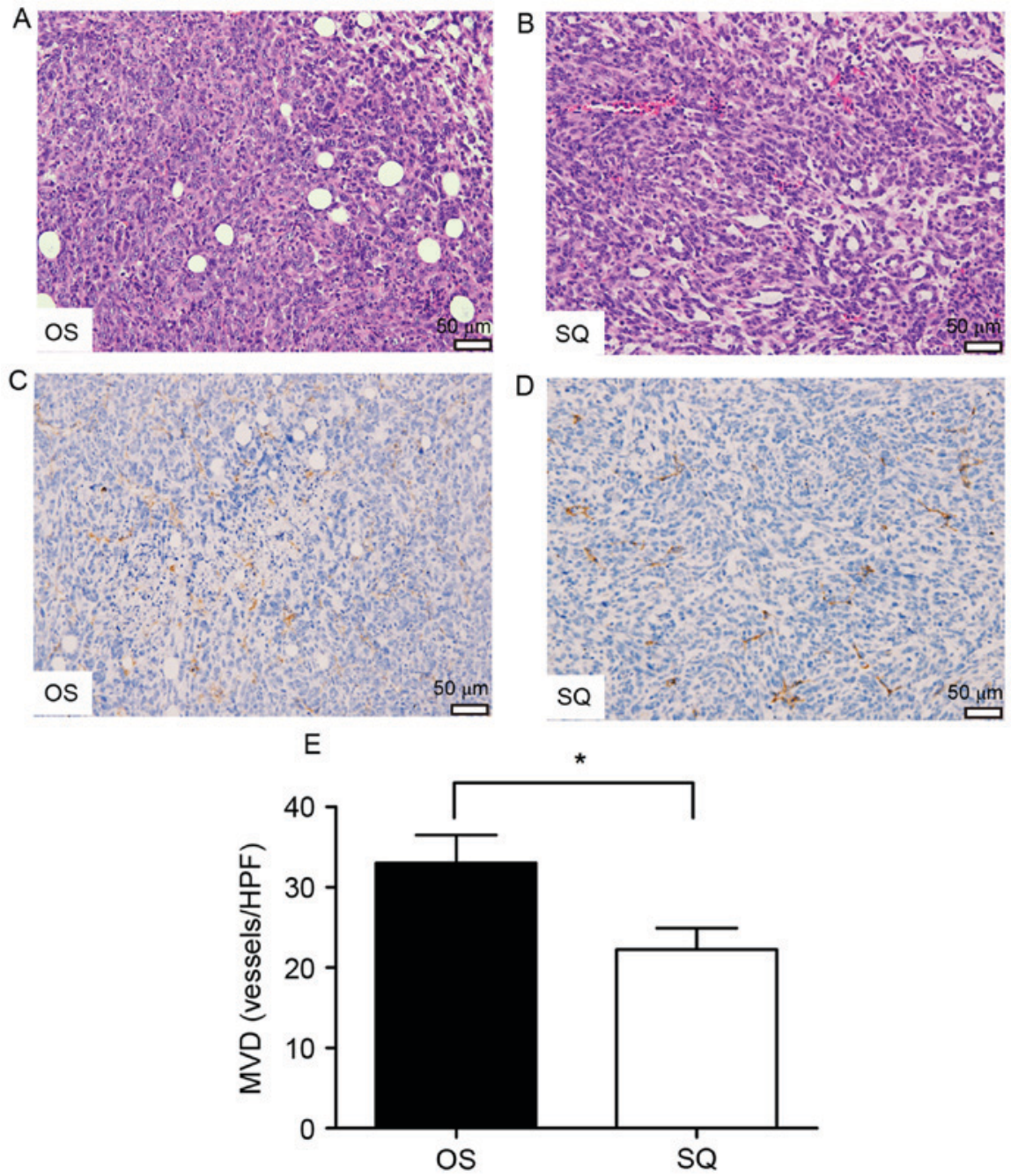

Figure 4.H\&E staining and immunohistochemical staining analysis of primary tumors (magnification, $\mathrm{x} 200$ ) with (A) OS and (B) SQ implantation. Representative CD31 staining of primary tumors with (C) OS implantation and (D) SQ implantation. Brownish staining indicated CD31 ${ }^{+}$vessels. (E) Quantification of MVD as numbers of $\mathrm{CD} 31^{+}$vessels/field (magnification, $\mathrm{x} 100 ;{ }^{*} \mathrm{P}<0.05$ ). $\mathrm{H} \& \mathrm{E}$, hematoxylin and eosin; $\mathrm{CD}$, cluster of differentiation; OS, orthotopic implantation; SQ, subcutaneous implantation; MVD, microvessel density; HPF, high-power field.

Cultured tumor cells or tumor tissue implantation were considered in the present study as there is currently no consensus for mouse modeling. It was hypothesized that tissue inoculation led to improved growth as they possessed stroma and extracellular matrix. Previous studies demonstrated that tissue inoculation resulted in decreased latency period and increased metastatic rate $(6,30,31)$. Despite this, results of the present study identified that 4T1-luc2 tissue inoculation caused a decreased tumor growth and a delay in tumor development. This is hypothesized to be a result of the increased potency of tissues in provoking an immune reaction, consequently causing increased necrosis or apoptosis $(5,32)$. An additional reason for the effects observed in the present study may be that the tissue disposal process damaged the stroma and extracellular matrix, which facilitated tumor growth. Thus, on the basis of the results of the present study, cultured 4T1-luc2 cells injected directly were considered to be a beneficial option.

The implantation site was subsequently compared using cultured 4T1-luc2 cells. Following inoculation of an equal number of cells using OS and SQ, tumor growth and metastasis were continuously determined by an in vivo imaging system and caliper measurement. The tumor activity and size of OS were increased compared with that of SQ during tumor development. OS primary tumors were of increased size and decreased variability. The results of the present study are consistent with previous studies of cancer cell growth and limitation of cell viability in SQ $(4,5,16,18,21,31,33)$. The decreased variation of OS tumor volume is favored for narrowing the gap within the group and for strengthening comparability between groups. As previous studies revealed, implantation into the mammary pad formed cancer cells with increased aggressive potential $(33,34)$. SQ primary tumors were observed to possess increased localization and were easy to remove in surgery. In comparison, OS primary tumors exhibited an increased invasive growth pattern. Subsequently, with $\sim 2$-fold primary tumor burden, OS models exhibited increased lung metastases. It is known that tumor growth and metastasis are dependent on the malignant biological behavior of cancer cell and on the tumor microenvironment where tumor cells were located $(35,36)$. The results of the present study identified an increased number of $\mathrm{CD} 1^{+}$vessels in OS tumors. Evidently, increased angiogenesis activity provided adequate blood and nutrients for tumor growth and metastasis in the OS model. In addition, adipocytes were observed in OS tumors which validates that adipose tissues and 

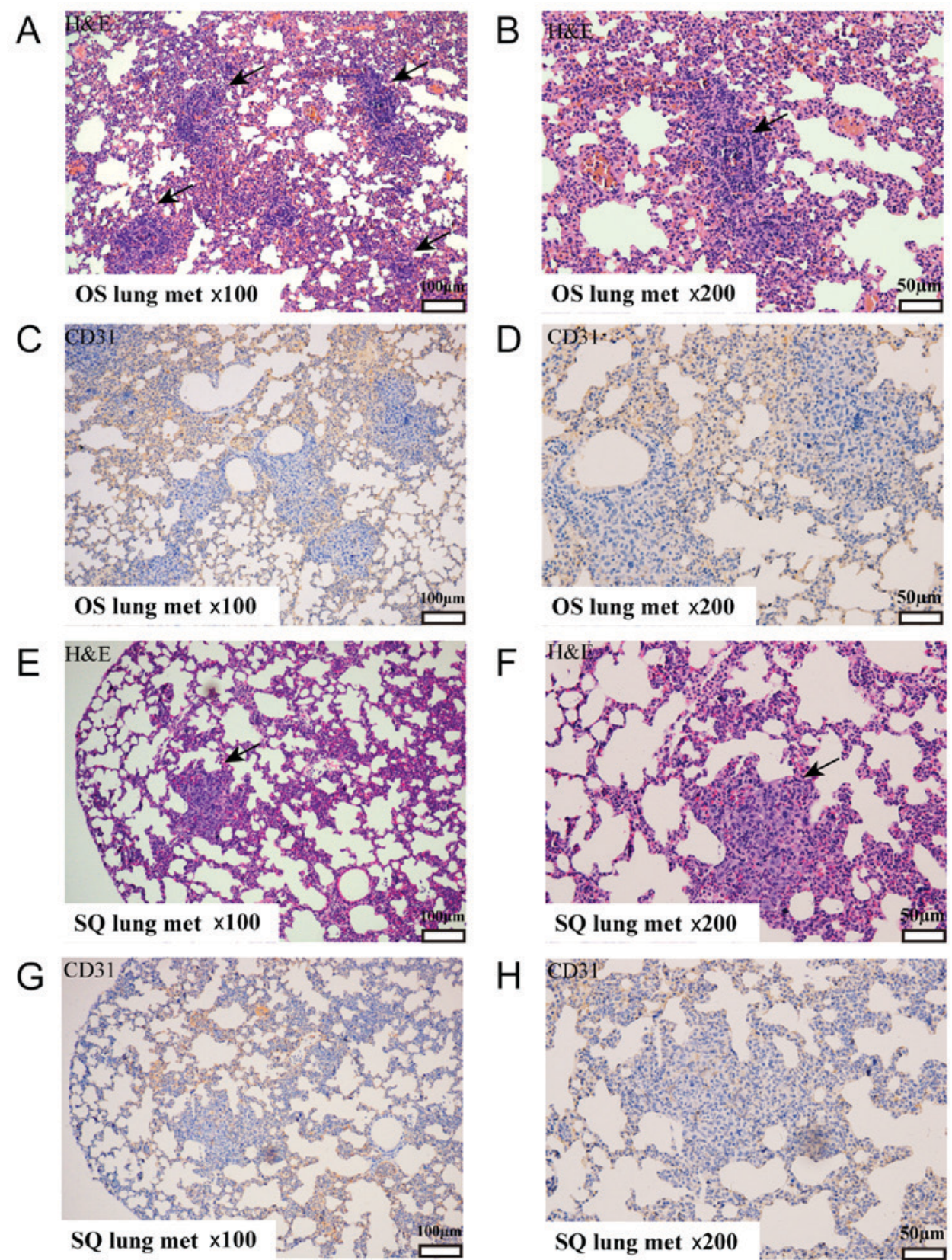

Figure 5. The metastatic lungs were fixed in buffered formalin, embedded in paraffin and stained. (A) OS section stained with H\&E; magnification, x100. (B) OS section stained with H\&E; magnification, x200. (C) OS section stained with anti-CD31 antibody; magnification, x100. (D) OS section stained with anti-CD31 antibody; magnification, x200. (E) SQ section stained with H\&E; magnification, x100. (F) SQ section stained with H\&E; magnification, x200. (G) SQ section stained with anti-CD31 antibody; magnification, x100. (H) SQ section stained with anti-CD31 antibody; magnification, x200. H\&E, Hematoxylin and eosin; OS, orthotopic implantation; SQ, subcutaneous implantation; CD, cluster of differentiation; met, metastasis.

adipocytes support tumorigenesis and metastasis, particularly in hormone-dependent cancers (37). The results of the present study indicate that OS models may be more suitable for studies involving tumor-microenvironment interaction. The common experimental protocol for the orthotopic breast cancer model is presented in Fig. 6. Although there are numerous advantages of OS, SQ implantation remains widely used (24) and is often combined with tail injection to observe metastasis.

In these murine models, the majority of studies have applied between $5 \times 10^{4}$ and $2 \times 10^{6} 4 \mathrm{~T} 1$ or $4 \mathrm{~T} 1$-luc 2 cells for inoculation into each mouse. The tumor volume reached $\geq 1,000 \mathrm{~mm}^{3}$ in 2 weeks, with an overall survival time of between 30 and 40 days $(22,33,38,39)$. In the present study, $1 \times 10^{4}$ cells were inoculated into each animal which resulted in a decreased volume of primary tumor $\left(<1,000 \mathrm{~mm}^{3}\right)$ and there was no mass mortality following primary tumor resection in SQ and OS models. The experiment was ended at day 56 due to ethical considerations. The results of the present study are considered to be of importance for designing experiments to improve mimicking the real clinical situation of cancer development, with less tumor burden.

The results of the present study identified that the various inocula (tissue or cell) and implantation site (OS or SQ) led to various rates of tumor growth and metastasis. OS generated increased malignant behavior compared with that of SQ. By understanding the characteristics of murine breast cancer 


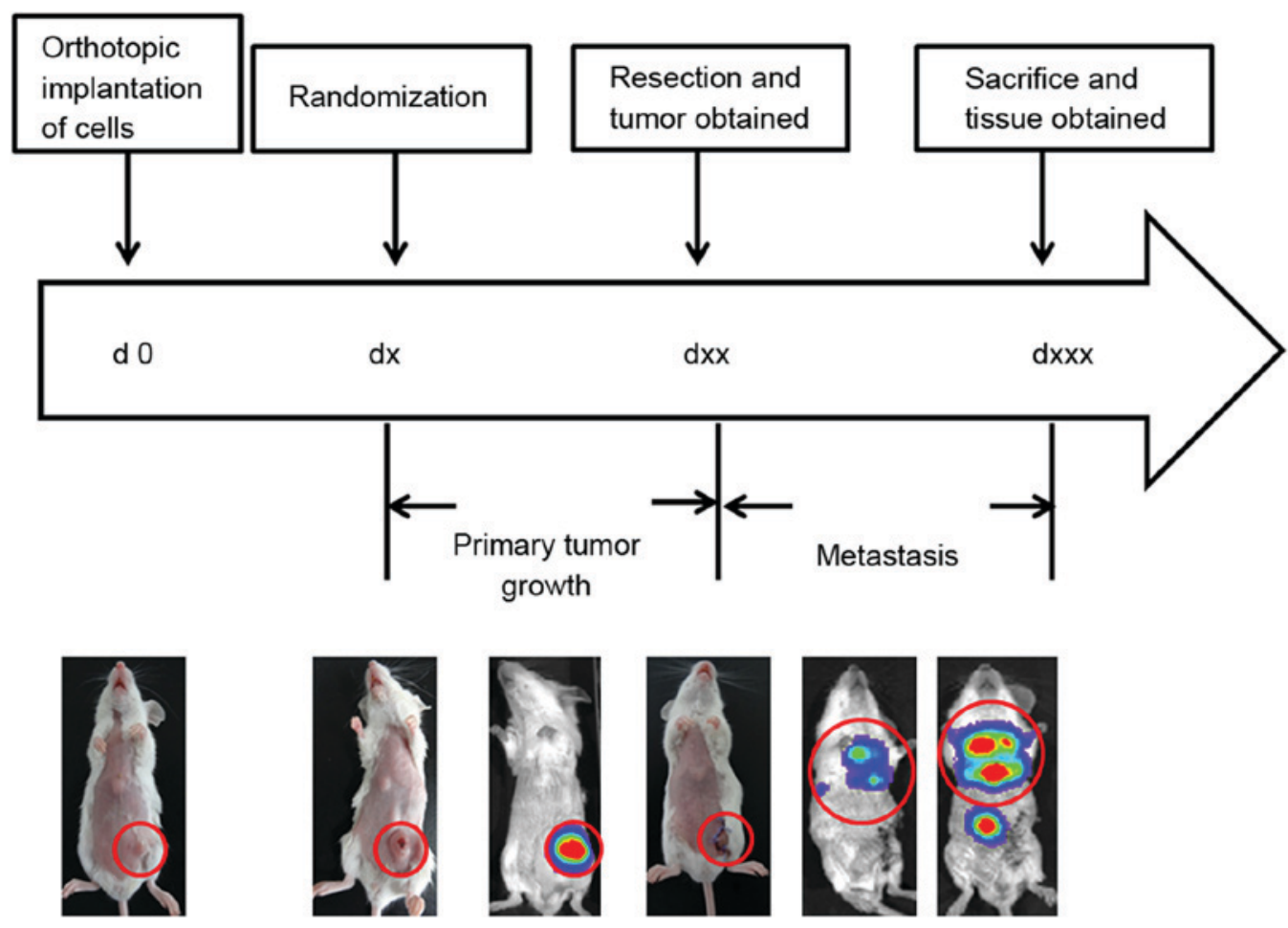

Figure 6. Experimental protocol for the orthotopic breast cancer model. Female BALB/c mice were injected with 4T1-luc2 cell suspension into the fourth mammary fat pad. Lung metastasis was able to be observed after resection of primary tumor. d, day(s).

models established by diverse methods, it is possible to select an appropriate model to use in further studies.

\section{Acknowledgements}

The authors would like to thank Yun-feng Zhao (Institute of Microbiology, Chinese Academy of Sciences, Beijing, China) for technical support with the animal experiments and Jin-Ping Li (Department of Medical Biochemistry and Microbiology, Uppsala University, Uppsala, Sweden) for manuscript revision.

\section{Funding}

The present study was funded by the National Natural Science Foundation of China (grant nos. 81673924 and 81373815), the Beijing Natural Science Foundation (grant no. 7162084), the Specialized Research Fund for the Doctoral Program of Higher Education of China (grant no. 20131107110014) and the Swedish Cancer Foundation (grant no. 150815).

\section{Availability of data and materials}

The datasets used and/or analyzed during the current study are available from the corresponding author on reasonable request.

\section{Authors' contributions}

YZ, GLZ, XS, KXC, GWY and MWY conducted the experiments. XMW and GLZ conceived and designed the experiments. CM and NN analyzed the data. YZ and GLZ wrote the manuscript.

\section{Ethics approval and consent to participate}

Animal experiments were conducted in accordance with the Provision and General Recommendation of Chinese Experimental Animals Administration Legislation and were approved by the Institution of Animal Care and Use Committee of Capital Medical University (ref. no. AEEI-2014-052).

\section{Consent for publication}

Not applicable.

\section{Competing interests}

The authors declare that they have no competing interests.

\section{References}

1. Torre LA, Bray F, Siegel RL, Ferlay J, Lortet-Tieulent J and Jemal A: Global cancer statistics, 2012. CA Cancer J Clin 65: $87-108,2015$

2. Shi XJ, Au WW, Wu KS, Chen LX and Lin K: Mortality characteristics and prediction of female breast cancer in China from 1991 to 2011. Asian Pac J Cancer Prev 15: 2785-2791, 2014.

3. Light DW: Global drug discovery: Europe is ahead. Health Aff (Millwood) 28: w969-w977, 2009.

4. Ottewell PD, Coleman RE and Holen I: From genetic abnormality to metastases: Murine models of breast cancer and their use in the development of anticancer therapies. Breast Cancer Res Treat 96: 101-113, 2006.

5. Rashid OM and Takabe K: Animal models for exploring the pharmacokinetics of breast cancer therapies. Expert Opin Drug Metab Toxicol 11: 221-230, 2015.

6. Wagner KU: Models of breast cancer: Quo vadis, animal modeling? Breast Cancer Res 6: 31-38, 2004. 
7. Donehower LA, Harvey M, Slagle BL, McArthur MJ, Montgomery CA Jr, Butel JS and Bradley A: Mice deficient for p53 are developmentally normal but susceptible to spontaneous tumours. Nature 356: 215-221, 1992.

8. Escobar Galvis ML, Jia J, Zhang X, Jastrebova N, Spillmann D, Gottfridsson E, van Kuppevelt TH, Zcharia E, Vlodavsky I, Lindahl U and Li JP: Transgenic or tumor-induced expression of heparanase upregulates sulfation of heparan sulfate. Nat Chem Biol 3: 773-778, 2007.

9. Matsui Y, Halter SA, Holt JT, Hogan BL and Coffey RJ: Development of mammary hyperplasia and neoplasia in MMTV-TGF alpha transgenic mice. Cell 61: 1147-1155, 1990.

10. Ursini-Siegel J, Schade B, Cardiff RD and Muller WJ: Insights from transgenic mouse models of ERBB2-induced breast cancer. Nat Rev Cancer 7: 389-397, 2007.

11. Menezes ME, Das SK, Emdad L, Windle JJ, Wang XY, Sarkar D and Fisher PB: Genetically engineered mice as experimental tools to dissect the critical events in breast cancer. Adv Cancer Res 121: 331-382, 2014.

12. Chakravarty G, Mathur A, Mallade P, Gerlach S, Willis J, Datta A, Srivastav S, Abdel-Mageed AB and Mondal D: Nelfinavir targets multiple drug resistance mechanisms to increase the efficacy of doxorubicin in MCF-7/Dox breast cancer cells. Biochimie 124: 53-64, 2016.

13. Li H, Pan GF, Jiang ZZ, Yang J, Sun LX and Zhang LY: Triptolide inhibits human breast cancer MCF-7 cell growth via downregulation of the ER $\alpha$-mediated signaling pathway. Acta Pharmacol Sin 36: 606-613, 2015.

14. Habu S, Fukui H, Shimamura K, Kasai M, Nagai Y, Okumura K and Tamaoki N: In vivo effects of anti-asialo GM1. I. Reduction of NK activity and enhancement of transplanted tumor growth in nude mice. J Immunol 127: 34-38, 1981.

15. Marangoni E, Vincent-Salomon A, Auger N, Degeorges A, Assayag F, de Cremoux P, de Plater L, Guyader C, De Pinieux G, Judde JG, et al: A new model of patient tumor-derived breast cancer xenografts for preclinical assays. Clin Cancer Res 13: 3989-3998, 2007.

16. Schuh JC: Trials, tribulations, and trends in tumor modeling in mice. Toxicol Pathol 32 (Suppl 1): S53-S66, 2004.

17. Talmadge JE, Singh RK, Fidler IJ and Raz A: Murine models to evaluate novel and conventional therapeutic strategies for cancer. Am J Pathol 170: 793-804, 2007.

18. Gravekamp C, Sypniewska R, Gauntt S, Tarango M, Price P and Reddick R: Behavior of metastatic and nonmetastatic breas tumors in old mice. Exp Biol Med (Maywood) 229: 665-675, 2004

19. Singh M, Ramos I, Asafu-Adjei D, Quispe-Tintaya W, Chandra D, Jahangir A, Zang X, Aggarwal BB and Gravekamp C: Curcumin improves the therapeutic efficacy of Listeria(at)-Mage-b vaccine in correlation with improved T-cell responses in blood of a triple-negative breast cancer model 4T1. Cancer Med 2: 571-582, 2013.

20. Takahashi K, Nagai N, Ogura K, Tsuneyama K, Saiki I, Irimura T and Hayakawa Y: Mammary tissue microenvironment determines $\mathrm{T}$ cell-dependent breast cancer-associated inflammation. Cancer Sci 106: 867-874, 2015.

21. Tao K, Fang M, Alroy J and Sahagian GG: Imagable 4T1 model for the study of late stage breast cancer. BMC Cancer 8: 228 , 2008.

22. Zhou H, Roy S, Cochran E, Zouaoui R, Chu CL, Duffner J, Zhao G, Smith S, Galcheva-Gargova Z, Karlgren J, et al: M402, a novel heparan sulfate mimetic, targets multiple pathways implicated in tumor progression and metastasis. PLoS One 6: e21106, 2011.

23. Lee HS, Ha AW and Kim WK: Effect of resveratrol on the metastasis of 4T1 mouse breast cancer cells in vitro and in vivo. Nutr Res Pract 6: 294-300, 2012

24. Mehta RR, Katta H, Kalra A, Patel R, Gupta A, Alimirah F, Murillo G, Peng X, Unni A, Muzzio M and Mehta RG: Efficacy and mechanism of action of Deguelin in suppressing metastasis of 4T1 cells. Clin Exp Metastasis 30: 855-866, 2013
25. Gibson-D'Ambrosio RE, Samuel M and D'Ambrosio SM: A method for isolating large numbers of viable disaggregated cells from various human tissues for cell culture establishment. In Vitro Cell Dev Biol 22: 529-534, 1986.

26. Weigand A, Boos AM, Tasbihi K, Beier JP, Dalton PD, Schrauder M, Horch RE, Beckmann MW, Strissel PL and Strick R: Selective isolation and characterization of primary cells from normal breast and tumors reveal plasticity of adipose derived stem cells. Breast Cancer Res 18: 32, 2016.

27. Weidner N, Semple JP, Welch WR and Folkman J: Tumor angiogenesis and metastasis-correlation in invasive breast carcinoma. N Engl J Med 324: 1-8, 1991.

28. Jordan VC: Proven value of translational research with appropriate animal models to advance breast cancer treatment and save lives: The tamoxifen tale. Br J Clin Pharmacol 79: 254-267, 2015.

29. Clarke R: Animal models of breast cancer: Their diversity and role in biomedical research. Breast Cancer Res Treat 39: 1-6, 1996.

30. Morioka CY, Saito S, Ohzawa K, Asano S, Hibino Y, Nakada Y, Kita KI and Watanabe A: Subcutaneously inoculated cells and implanted pancreatic cancer tissue show different patterns of metastases in Syrian golden hamsters. JOP 1: 183-190, 2000.

31. Rao Q, You A, Guo Z, Zuo B, Gao X, Zhang T, Du Z, Wu C and Yin H: Intrahepatic tissue implantation represents a favorable approach for establishing orthotopic transplantation hepatocellular carcinoma mouse models. PLoS One 11: e148263, 2016.

32. de la Cruz-Merino L, Barco-Sánchez A, Henao Carrasco F, Nogales Fernández E, Vallejo Benítez A, Brugal Molina J, Martínez Peinado A, Grueso López A, Ruiz Borrego M, Codes Manuel de Villena M, et al: New insights into the role of the immune microenvironment in breast carcinoma. Clin Dev Immunol 2013: 785317, 2013.

33. Rashid OM, Nagahashi M, Ramachandran S, Dumur C, Schaum J, Yamada A, Terracina KP, Milstien S, Spiegel S and Takabe K: An improved syngeneic orthotopic murine model of human breast cancer progression. Breast Cancer Res Treat 147: 501-512, 2014.

34. Rashid OM, Nagahashi M, Ramachandran S, Dumur CI, Schaum JC, Yamada A, Aoyagi T, Milstien S, Spiegel S and Takabe K: Is tail vein injection a relevant breast cancer lung metastasis model? J Thorac Dis 5: 385-392, 2013.

35. Bibby MC: Orthotopic models of cancer for preclinical drug evaluation: Advantages and disadvantages. Eur J Cancer 40: $852-857,2004$

36. Fidler IJ: The pathogenesis of cancer metastasis: The 'seed and soil' hypothesis revisited. Nat Rev Cancer 3: 453-458, 2003.

37. Nieman KM, Romero IL, Van Houten B and Lengyel E: Adipose tissue and adipocytes support tumorigenesis and metastasis. Biochim Biophys Acta 1831: 1533-1541, 2013.

38. Hammond E, Brandt R and Dredge K: PG545, a heparan sulfate mimetic, reduces heparanase expression in vivo, blocks spontaneous metastases and enhances overall survival in the 4T1 breast carcinoma model. PLoS One 7: e52175, 2012.

39. Kim JB, Urban K, Cochran E, Lee S, Ang A, Rice B, Bata A, Campbell K, Coffee R, Gorodinsky A, et al: Non-invasive detection of a small number of bioluminescent cancer cells in vivo. PLoS One 5: e9364, 2010.

This work is licensed under a Creative Commons Attribution-NonCommercial-NoDerivatives 4.0 International (CC BY-NC-ND 4.0) License. 